\title{
Descripción del uso de la tapia pisada con tierra estabilizada en Australia
}

\section{The stabilized rammed earth building technique and its use in Australia}

\author{
Rodrigo Amaral do Prado Rocha* y Pedro Henryque Melo de Oliveira**
}

\section{Resumen}

La técnica constructiva de tapia pisada que se realiza desde hace siglos en diversos países, está presente en Australia hace décadas. En este país, las construcciones en tapia pisada ya presentan avances técnicos importantes para la ejecución de obras en tapia, siendo destacados en este estudio algunos avances relacionados con detalles constructivos: rasgos del material, cuidados en la ejecución, montaje de las cimbras y patologías de la construcción, que pueden ser propias o investigadas para la tapia pisada convencional. El presente artículo tiene por objetivo informar y actualizar a los profesionales y estudiantes sobre una tecnología en desarrollo que hace uso de la tierra como su principal material de construcción, a través de información relevante para la producción contemporánea de construcciones en tierra. Se pretende compartir experiencias constructivas y proyectuales que puedan ser útiles en la ampliación del conocimiento sobre los límites y las necesidades constructivas, para -de este modo- contribuir a la investigación sobre su cadena productiva, desde el abastecimiento del material hasta su mantenimiento. A través de una relativa acumulación de conocimiento producida hasta hoy sobre la técnica en cuestión, es posible romper con algunos preconceptos o distorsiones sobre el uso de la tierra como material de construcción civil. Parte de ese conocimiento y experiencia es aquí compartido con la intención de contribuir a la divulgación acerca del uso de la tapia pisada contemporánea.

Palabras clave: construcción en tierra, tapia mecanizada, arquitectura contemporánea australiana, desarrollo de técnica constructiva.

\section{Abstract}

The building technique known as rammed earth has been used in Australian civil construction for decades. This practice, used in various regions of Australia, has brought with it impor-

Fecha de recepción: 19 de mayo de 2019 - Fecha de aceptación: 16 de julio de 2019

\footnotetext{
*Arquitecto y urbanista. Trabaja como arquitecto proyectista en Olnee Constructions en Melbourne, Australia. Correo electrónico: arqrodrigoamaral@gmail.com

***Arquitecto y urbanista. Magíster en Arquitectura y Urbanismo de la Universidad Federal de Uberlândia, UFU, Brasil. Correo electrónico: pedrohenryque@hotmail.com
} 
tant technical improvements to the execution of Stabilized Rammed Earth (SRE) buildings. This study highlights some of those advances in terms of construction details, composition, special cares during execution, the use of formwork, and construction pathologies, however, these can be considered and incorporated into the traditional (unstabilized) rammed earth technique. This article aims to inform and update professionals and students about the evolving technology of using earth as the main building material, providing relevant information about its contemporary production. It aims to share design and building insights that may enrich the knowledge around the limits and needs of the SRE, and to contribute to the research of its productive chain, from the supply of raw materials to maintenance. By acquiring the knowledge produced so far regarding this technique, it is possible to break free from preconceptions and misconceptions about the earth as a building material. Part of this knowledge is hereby analyzed and shared, to contribute of the dissemination of the use of the Stabilised Rammed Earth technique.

Keywords: earth building, stabilized rammed earth, contemporary Australian architecture, development of building techniques.

\section{Introducción}

Según algunos estudiosos (Rael, 1971; Dethier, 1986; Eires y jalali., 2008; Torgal, Eires y Jalali., 2009) casi la mitad de la población mundial vive en construcciones hechas con tierra. Actualmente, construir con tierra va al encuentro de cuestiones fundamentales para la práctica de una arquitectura "responsable" o mínimamente "consciente" de su impacto ambiental, teniendo en cuenta su participación en la alteración del medio ambiente, la contaminación y el consumo de recursos naturales en general, causados por la industria de la construcción. De acuerdo con John, Silva y Agopyan (2001), "la industria de la construcción, así como sus productos consumen aproximadamente $40 \%$ de la energía y los recursos naturales y generan 40\% de los residuos producidos por todo el conjunto de actividades humanas, más se puede alcanzar hasta el 75\%, como en el caso de los EUA" (p. 92). La técnica aquí abordada, tapia pisada, así como otras técnicas con tierra, puede contribuir a mitigar los efectos nocivos de la construcción civil.

Este texto trata sobre las experiencias y aplicaciones actuales de la tapia pisada en Australia; más precisamente, se trata de una descripción del trabajo desarrollado en una empresa en Melbourne, Estado de Victoria. Después de abordar la diferencia entre la práctica mecanizada australiana de una versión más "original" o tradicional de la tapia pisada, el artículo comenta sobre algunas particularidades y características del proyecto y de la ejecución de esta técnica.

La vasta producción actual de construcciones con tierra en Australia tiene sus orígenes en la segunda mitad del siglo XX, cuando la tapia se vuelve una técnica extremadamente popular en innumerables regiones de este país posiblemente estimulada, o al menos amparada, por la legislación referente al uso de la tierra en la construcción civil. Hay un pionerismo en lo que se refiere a normas constructivas para tierra, pues según Torgal et al., Australia es:

uno de los primeros países que tiene una reglamentación específica sobre la construcción en tierra. La misma fue publicada en 1952 por la Commonwealth Scientific and Industrial Research Organization (CSIRO) con la designación de "Bulletin 5", habiendo sido objeto de sucesivas reediciones en 1976, 1981, 1987 y 1992. Este reglamento representó hasta hace muy poco las bases para el proyecto de la construcción en tierra, en las variantes de adobe, tapia pisada y BTC. Reconociendo la necesidad de un documento más completo y más actual que el "Bulletin 5", se publicó en 2002 el Manual de construcción en tierra Australiano (p. 25).

Esta reciente producción arquitectónica con tierra ha prosperado y se encuentra actualmente en boga; posiblemente por la falta de historia del uso 
de la tierra en construcciones, no se han creado preconceptos ni resistencias culturales con relación a este material, a diferencia de lo que sucede en otros países donde sí se relaciona la tapia con el colonialismo, el patrimonio histórico, lo arcaico, lo premoderno, con edificaciones precarias y típicas de personas de bajos ingresos. En este sentido, agrega Braga Fernandes (2013):

A pesar de que existen otras técnicas en tierra en este país, como el adobe, los bloques cortados y las técnicas mixtas, es un hecho que la tapia pisada predomina en la arquitectura contemporánea australiana, resultando en uno de los países que más invirtió en la reglamentación, en la investigación universitaria y en la implementación actual de numerosos proyectos (p. 20).

\section{Sobre la producción de tapia pisada hecha en Australia}

Para iniciar la discusión sobre la producción de la tapia en Australia se esboza la cadena productiva en que está inserta, que aquí se divide de manera didáctica en tres partes: 1) obtención del material, 2) fabricación de la cimbra y 3) experiencia de los técnicos y constructores. Sobre la obtención del material de construcción es importante puntuar que no se restringe sólo a la tierra presente en el cantero de obras. En Australia, los proveedores de suelo y agregados de construcción, generalmente empresas con función de suministrar material para pavimentación de vías y carreteras, son responsables también de proveer material para la producción de la tapia. Estas empresas proporcionan diferentes clases de agregados, generalmente tipos de grava, sedimentos de rocas, arcilla arenosa y arcillosa con diversas granulometrías, además de triturar y reutilizar hormigón y ladrillos macizos. La diversidad y disponibilidad de suelos y agregados son fundamentales para la producción y difusión de las paredes de tapia.

Los otros dos puntos están relacionados de manera inextricable ya que fue a partir de la práctica de constructores y técnicos que hubo el desarrollo del sistema constructivo, resultando en la alteración de la fabricación y concepción de las cimbras, componente central para la producción de paredes en tapia pisada. Actualmente, muchos arquitectos e ingenieros australianos ya saben cómo proyectar y calcular para el uso de la tapia, lo que fomenta y amplía la producción y, consecuentemente, posibilita que más constructores sepan cómo trabajar y más productos y equipos relacionados con la producción de la tapia pueden mejorarse para la optimización de la producción de esta técnica, lo cual es una importante contribución de la práctica australiana.

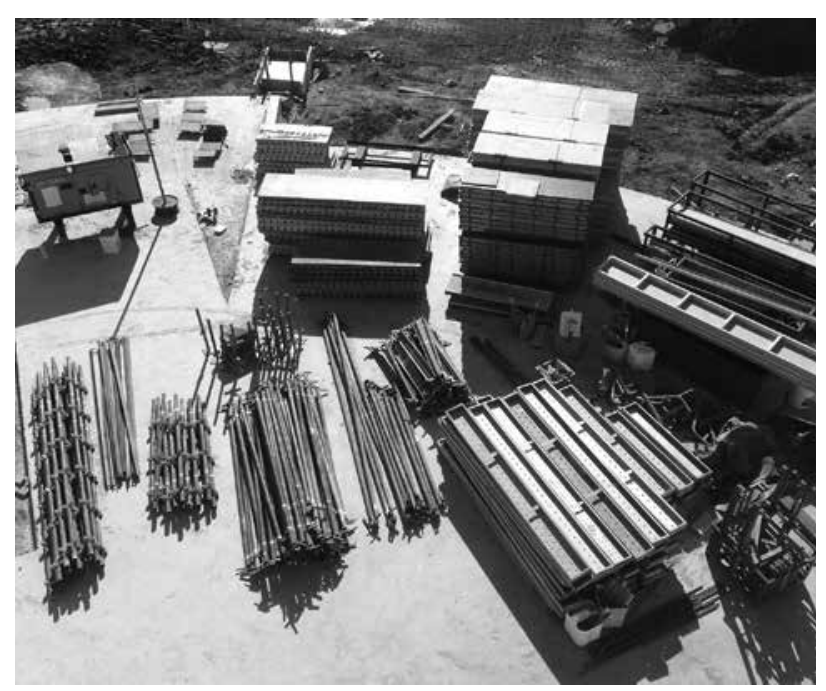

Figura 1. Equipos necesarios para la ejecución de la tierra estabilizada compactada, 2014. Fuente: Oliver Petrovic.

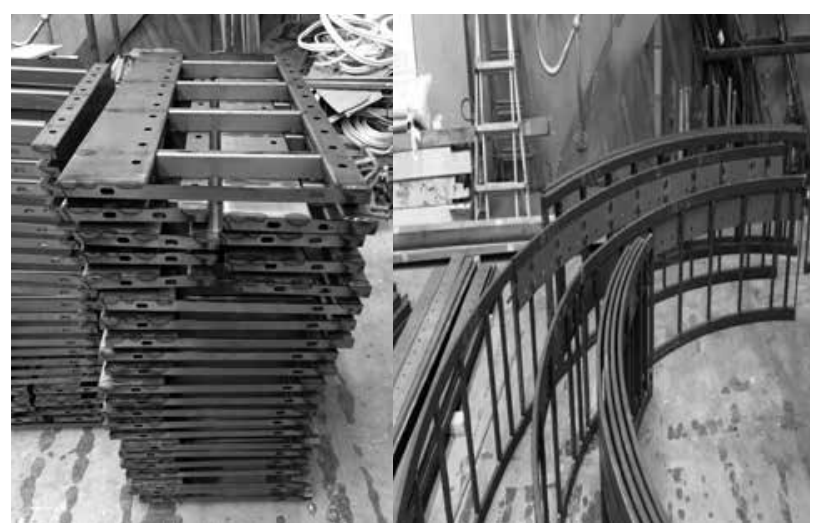

Figura 2. Ejemplos de moldes metálicos planos y curvas de la constructora Olnee, 2017. Fuente: elaboración propia. 
Después de observar la obtención del material y ponderar sintéticamente sobre su ejecución, es importante concentrarse en las especificaciones de las formaletas usadas para la ejecución de las paredes, pues éstas son diferentes de las destinadas a la construcción en concreto armado. Generalmente las formaletas en cuestión son producidas por los propios constructores de tapia, todas en madera con un esqueleto metálico, como se verá de manera detallada a continuación. En las Figuras 1 y 2 tenemos una imagen ilustrativa de los principales instrumentos y herramientas necesarios para la realización de una obra en tapia.

\section{Características del método australiano}

El método de construcción australiano de la tapia pisada se viene desarrollando a través de la mecanización de la técnica tradicional, logrando a lo largo de décadas de aplicación algunos avances importantes. Se destacan dos puntos principales: la estabilización de la tierra y la fabricación de las formaletas, sin contar la compactación mecanizada que confiere efectividad y adecuación a la producción contemporánea.

El procedimiento tradicional para la ejecución de obras en tierra compactada se realiza a partir de moldes de madera trabados por guías verticales externas, fijas o móviles, rellenadas con una mezcla de suelo con estabilizantes naturales, la arcilla ya presente en el suelo utilizado o con la cal.

La práctica australiana además de utilizar cemento como principal estabilizante, usa trabamiento horizontal de las molduras metálicas, no requiriendo del uso de guías externas para su trabamiento. Esto permite al constructor apilar el material de fuera sobre plataformas metálicas apoyadas en cantoneras también metálicas engastadas en las propias formaletas. Esta alteración trae importantes cambios para el sistema constructivo de tapia pisada pues confiere agilidad al proceso de

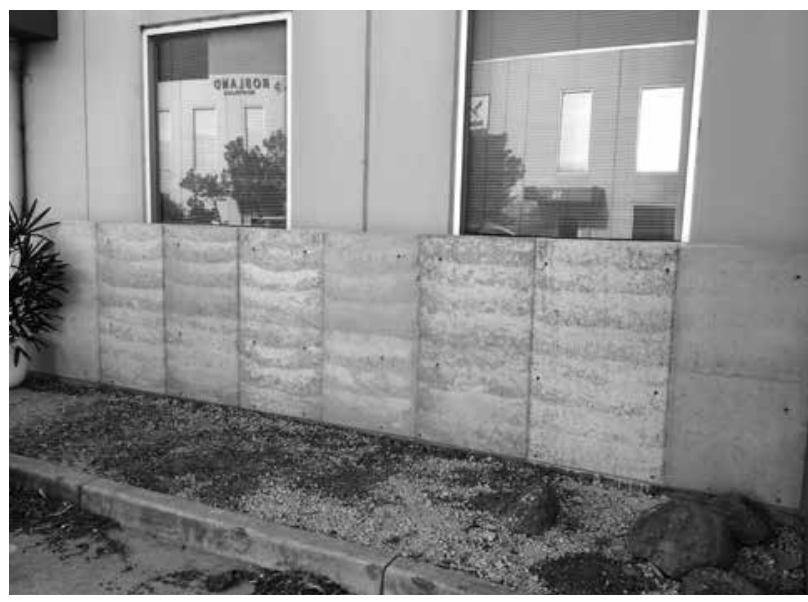

Figura 3. Colores y texturas de diferentes tapias pisadas en la fachada de la constructora Olnee Constructions, 2015. Fuente: elaboración propia.

construcción, posibilitando un trabajo continuo y eficiente. Otra alteración, en términos estéticos y económicos, está en el hecho de que con esas molduras las paredes pueden tener hasta $20 \mathrm{~cm}$ de espesor, más esbeltas que las realizadas por la técnica tradicional, generalmente con un mínimo de $40 \mathrm{~cm}$.

También hay cambios en el diseño y el rendimiento estructural de la pared. En la técnica tradicional la tierra apilada se mantiene por su peso propio, pues la resistencia de la pared está en la gran inercia de su masa que resiste sólo a los esfuerzos de compresión, siendo así autoportante. En la práctica contemporánea la pared de tapia puede funcionar de otra manera, análoga a una estructura de hormigón armado, pasa a ser resistente a esfuerzos de tracción, debido a la estabilización con cemento y con armazón en barras de acero, como ilustra la Figura 4 de la ejecución de una torre de 12 metros de altura.

\section{a) Fabricación y utilización de la formaleta/ encofrado/cimbra}

La formaleta/encofrado utilizada predominantemente en Australia fue desarrollada en la ciudad de Perth, en el estado de Australia Occidental. Después de varios años utilizando formaletas con 
estructura de madera con lámina de plywood/ contrachapado/triplay, con herramientas $y$ equipos específicos (columnas de traba, tornillos, espaciadores, andamios/obra falsa y otros más), los constructores verificaron que podían aumentar el rendimiento de la producción de las paredes a partir de la utilización de formaletas con estructura metálica y lámina de contrachapado. Esas formaletas tienen dimensiones fijas entre 30 - $60 \mathrm{~cm}$ de altura y 240,180, 120 o $90 \mathrm{~cm}$ de ancho, pesando de 10 a $30 \mathrm{~kg}$.

Las formaletas son estructuradas por piezas prefabricadas metálicas soldadas, un patrón desarrollado a partir de la necesidad de personalizar/ adaptar las formaletas (a cada proyecto) como muestra la Figura 3. Una lámina de contrachapado es atornillada al esqueleto metálico y luego a la parte superior e inferior de la formaleta le son realizados agujeros de $1,5 \mathrm{~cm}$ de diámetro a cada $15 \mathrm{~cm}$ sobre la medida de mayor extensión. Esta cantidad de perforaciones facilita el proceso de montaje de las formaletas, sin embargo, al desencofrar los agujeros quedan evidentes sobre la cara acabada de las paredes. Una capa de resina de fibra de vidrio es aplicada sobre la superficie del contrachapado garantizando así su impermeabilización y por consiguiente una mayor durabilidad de la formaleta, ya que además de proteger la madera que entra en contacto con la tierra, facilita la etapa final de desencofrado y asegura la calidad de la superficie de la pared terminada.

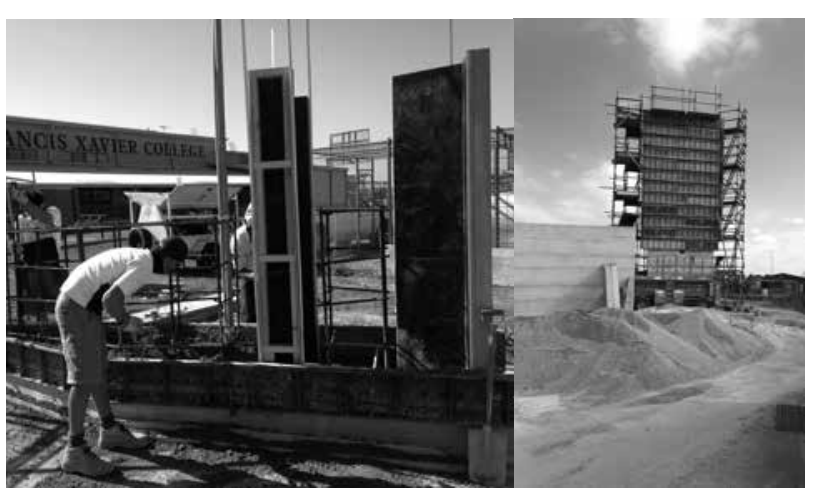

Figura 4. Ejemplo de ejecución de torre de 12 metros de altura de tapia armada. Fuente: Oliver Petrovic.

\section{b) Mezcla y masa ideales, tipos de tierra y uso de cemento y agua}

La estabilización de la tapia pisada con el uso de cemento posibilita la aplicación en ambientes externos, así como elimina la necesidad de revocar o revestir la pared. De acuerdo con Ciancio y Beckett (2015) la cantidad aceptable es de $5 \%$ a $15 \%$ de cemento, en términos de garantía de esfuerzos estructurales, longevidad, estabilización y capacidad de soportar cargas en compresión. Según Dobson (2015) un 10\% de la unidad de mezcla es lo ideal para garantizar una resistencia a la compresión de 2,5 a $5 \mathrm{MPa}(25$ a $50 \mathrm{kgf} / \mathrm{cm}^{2}$ ), lo suficiente para utilizar la tapia como mampostería de cerramiento, de acuerdo con Middleton (1992).

Los tipos de suelos utilizados en Australia varían de acuerdo con las necesidades del proyecto y del uso de las paredes. Según Middleton (1992) normalmente se utilizan uno o dos tipos de suelos, siendo éstos con máximo de $2 \%$ de materia orgánica, libre de hojas y astillas, entre 5 a $20 \%$ de arcilla, incluyendo barro y limo, mínimo con $20 \%$ de arena y piedras no superiores a $40 \mathrm{~mm}$ y un contenido de humedad entre 4 a 12\%. En algunos casos también se utiliza concreto triturado, así como ladrillos macizos triturados como agregados, materiales provenientes de desperdicios y restos de construcciones convencionales.

Para la mezcla ideal de la masa se utiliza un mini cargador que revuelve los materiales y garantiza que éstos sean bien mezclados, ya que debido a su baja humedad, la concretadora o mezclador no consiguen un resultado adecuado, pues parte de la masa es retenida y se pega a las paredes del aparato; además de agilizar el trabajo de poner la mezcla en el interior de las formaletas hasta a $3 \mathrm{~m}$ de altura, la altura máxima a la que el mini cargador alcanza, agiliza la ejecución de la pared, como muestra la Figura 5.

Se añaden aditivos a la mezcla con el fin de elevar la durabilidad de la pared. Se sugiere el uso del 


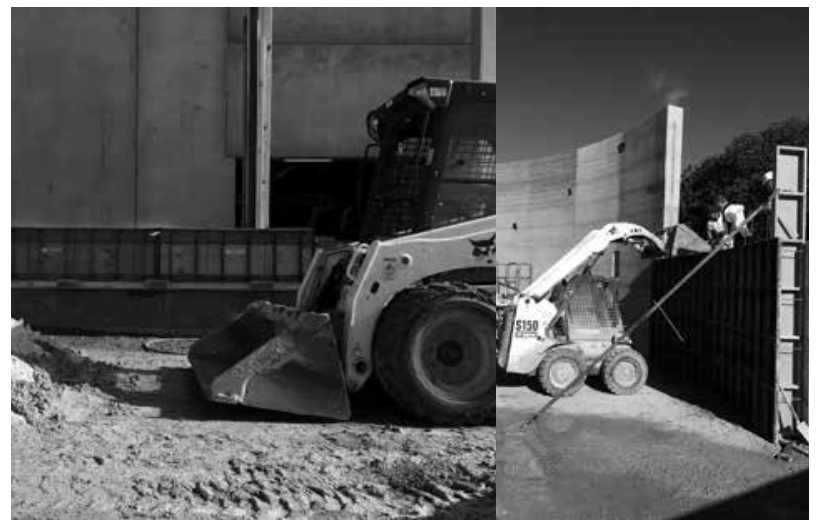

Figura 5. Las etapas de mezcla y relleno de las cimbras. A la izquierda la mini cargadora a punto para iniciar la mezcla del material de la balda. La imagen de la derecha muestra la misma alzando la mezcla para la ejecución de la pared. Fuente: Oliver Petrovic y Rodrigo Rocha.

$1 \%$ de plasticure, un producto desarrollado para paredes monolíticas en suelo-cemento. Al ser adicionado a la mezcla de tapia el plasticure garantiza la repelencia al agua, sal y moho, además de reforzar la estructura. Ese producto también es utilizado para vaciar slumps de concreto, o sea, para concretos de baja consistencia y su función es garantizar la resistencia a largo plazo contra la intemperie y el deterioro. Después de desencofrar las paredes, a las caras externas de las mismas se les aplica un sellante incoloro repelente al agua, capaz de penetrar en los capilares y asegurar que el sustrato se mantenga impermeable. La reducción de la absorción del agua, disminuirá la eflorescencia y otros problemas relacionados con el agua que son responsables de la mayor parte de la degradación en estructuras de tapia. El fabricante garantiza que la apariencia de los sustratos y la permeabilidad al vapor no son alteradas significativamente.

\section{c) Detalles de proyecto}

Conocer las posibilidades y saber proyectar la tapia es esencial para un proyecto adecuado a las necesidades y las capacidades de la técnica, que se asemeja al concreto armado, pues se trata de paredes autoportantes con propiedades estructurales suficientes para soportar cargas de compresión. De acuerdo con Ciancio y Beckett (2015), la tapia alcanza un rango desde $1 \mathrm{MPa}$ $\left(10 \mathrm{kgf} / \mathrm{cm}^{2}\right)$ hasta $47 \mathrm{MPa}\left(470 \mathrm{kgf} / \mathrm{cm}^{2}\right)$ de resistencia a la compresión, variación dada por la granulometría de los agregados, la presión de compactación y las cantidades de cemento y de agua.

Para ejecutar chimeneas $\mathrm{u}$ hornos en tapia, se utiliza una formaleta metálica con la forma deseada dentro de las paredes y se construye una pared maciza debajo, alrededor y encima de la formaleta metálica. El entubado de la salida/ tiro debe ser centrada y terminar encima de la superficie final de la pared. Al acabado del tope de la pared en casos de obra externa y expuesta al sol y a la lluvia, se le debe aplicar una mezcla de plasticure y agua, o como es ejecutado en Brasil y otros países en donde la última hilada de la pared tiene una mayor cantidad de cemento.

Con un proyecto ejecutivo finalizado es posible elaborar las instalaciones eléctricas e hidráulicas dentro de las paredes; sin embargo, cualquier alteración futura se torna inviable, como muestra la Figura 6. Las uniones con vigas y columnas estructurales posibilitan a la tapia interactuar con otros materiales como acero y madera, así como con otras técnicas constructivas en el caso del concreto armado, conforme ilustra la Figura 7.

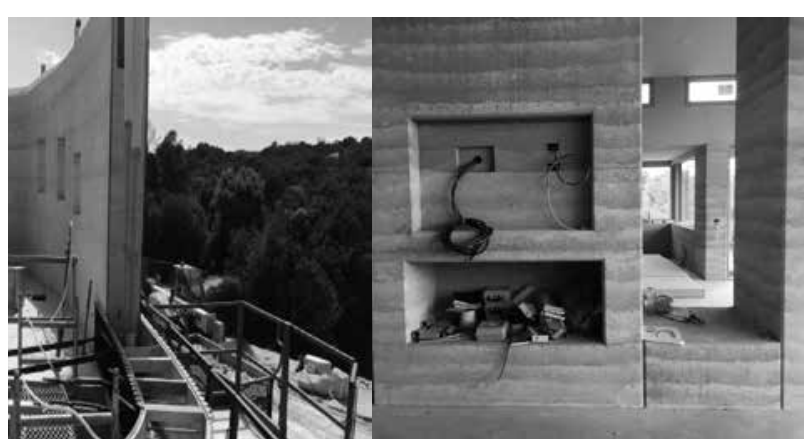

Figura 6. Ejemplos de detalles de instalación hidráulica embutida en pared curva a izquierda, instalación eléctrica embutida en residencia en Australia a la derecha. Fuente: Oliver Petrovic y Rodrigo Rocha. 


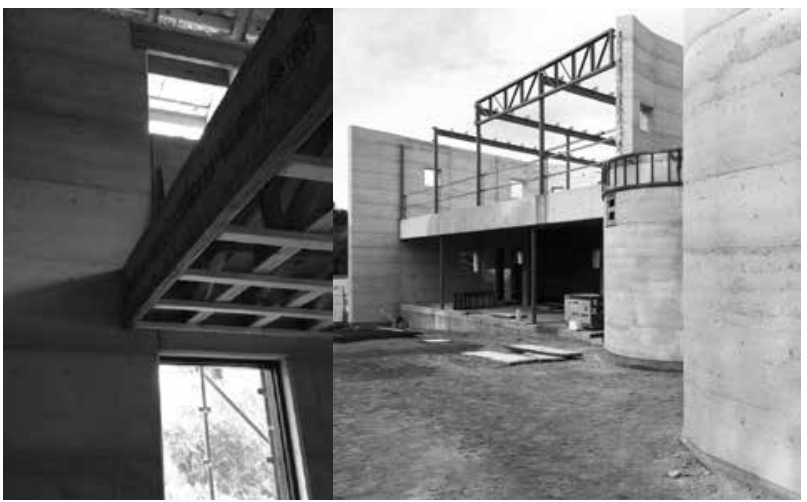

Figura 7. Ejemplos de conexiones de suelo de madera, viga de hormigón y viga metálica para cobertura. Fuente: Oliver Petrovic y Rodrigo Rocha.

\section{d) Detalles de ejecución}

La ejecución puede ser entendida como simple y es posible describirla de manera resumida por un sistema constructivo que involucra sólo cuatro constructores en el cantero: dos profesionales con experiencia en carpintería, albañilería y hormigón armado, y los otros dos pueden ser aprendices. Se hace necesario resaltar la importancia de la etapa de proyecto que define la paginación de las cimbras y distancia entre juntas de dilatación, además de resolver detalles arquitectónicos, conexiones estructurales, eléctricas e hidráulicas. De manera simplificada, la ejecución de la tapia puede ser descrita en dos etapas. En la primera se realiza el montaje de las molduras para que todo el material de relleno se coloque en capas, que serán apiladas hasta una altura de 15 a 20 centímetros sucesivamente, hasta alcanzar la altura final de la pared; se percibe que la altura de las capas define la cantidad de líneas de compactación, evidentes en la cara de la pared, como ilustra la Figura 3. La segunda etapa ocurre al día siguiente cuando se realiza la retirada de las cimbras, o la desforma, que marca el inicio del proceso de curación de la pared. Después de esta última etapa no hay necesidad de hacer ningún acabado o impermeabilización de paredes, a pesar de ser recurrente la aplicación de dos capas de hidrofugante transparente en sus caras acabadas.

$\mathrm{Al}$ iniciar la construcción de una pared se coloca una masa fuerte y espesa de cemento y agua en el bloque de la cimentación, para impermeabilizar y funcionar como transición de superficies, así como para aumentar la estabilidad de la pared. Para ejecutar vigas en tapia se utilizan perfiles metálicos en " $\mathrm{T}$ " conectados a columnas metálicas dentro de la pared. Se sugiere revestir la columna metálica con material con propiedades de dilatación, como espuma de EPDM expandido para hacer la transición de movimientos entre la estructura metálica y la pared. Para ejecutar la pared en una parte encima de un perfil metálico se monta la formaleta sobre vigas de madera atornilladas a la pared temporalmente, además de columnas en madera hechas en sitio como soporte de la viga hasta el momento del desencofrado.

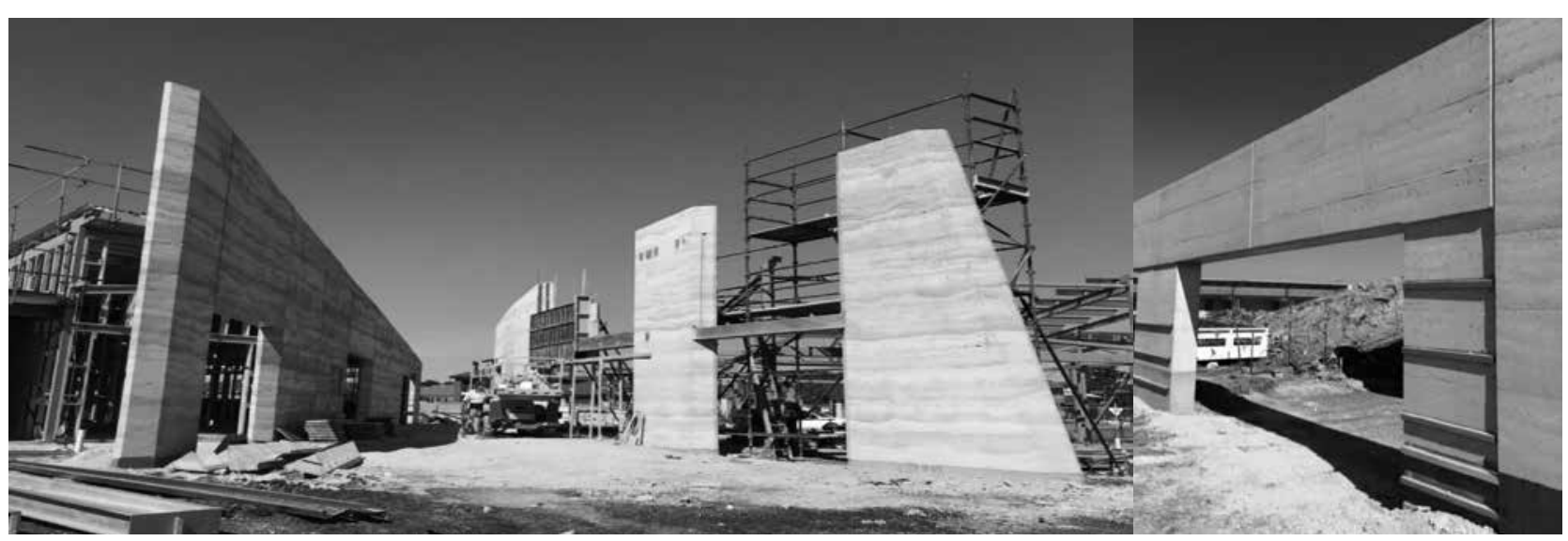

Figura 8. Ejecución de tapia pisada sobre viga metálica en “T” y columnas metálicas embutidas. Fuente: Oliver Petrovic y Rodrigo Rocha. 


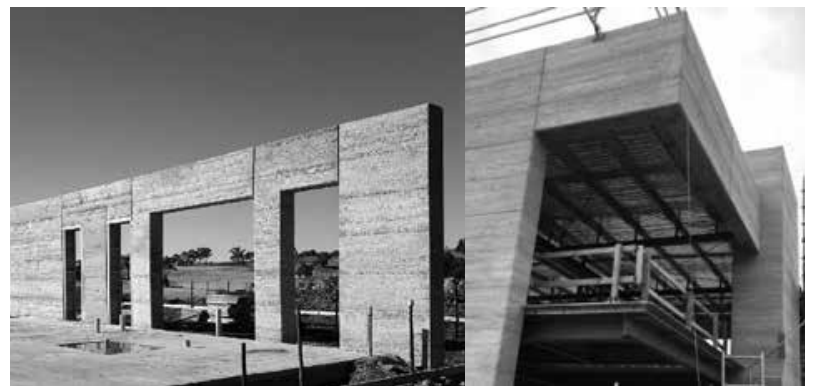

Figura 9. Ejemplos de ejecución de vigas de tapia por encima de vigas metálicas en “T”. Fuente: Oliver Petrovic y Rodrigo Rocha.

La utilización de un perfil metálico en " $\mathrm{T}$ ” posibilita vanos de hasta $10 \mathrm{~m}$ (Figuras 8 y 9). Para vanos hasta $4 \mathrm{~m}$ se sugiere utilizar apenas dos varillas/barras de acero de $12 \mathrm{~mm}$ a cada $20 \mathrm{~cm}$ en la horizontal, conectando las paredes laterales y soportando el movimiento de tracción de la viga.

El tiempo de curado de la pared va a depender de las cantidades de cemento y arcilla contenidas en la mezcla, así como de las condiciones climáticas locales. Algunos estudios comprueban que la tapia de pilón no estabilizada puede llevar hasta 2 años para curar y finalizar la retracción; sin embargo con el uso del cemento este tiempo disminuye hasta llegar a 30 días. Dependiendo de la cantidad de agua en la mezcla, se sugiere que en localidades con épocas de temperaturas elevadas y humedad relativa del aire baja, se deben humedecer las paredes en los días siguientes al desencofrado para que no ocurran retracciones rápidas y/o bruscas, lo que podría desencadenar fisuras y agrietamientos en la pared en función de la dilatación por reacción química entre los componentes de la mezcla.

Al momento de cubrir fisuras o imperfecciones en la pared se recomienda esperar al curado de las paredes para así posteriormente realizar alguna corrección. Es importante resaltar la atención que se debe prestar en utilizar las mismas proporciones para la mezcla de la pared para que no ocurra alteración de color o de textura en la superficie corregida. Después del curado en algunos casos se aplica sellador incoloro repelente al agua en las caras externas de la pared.

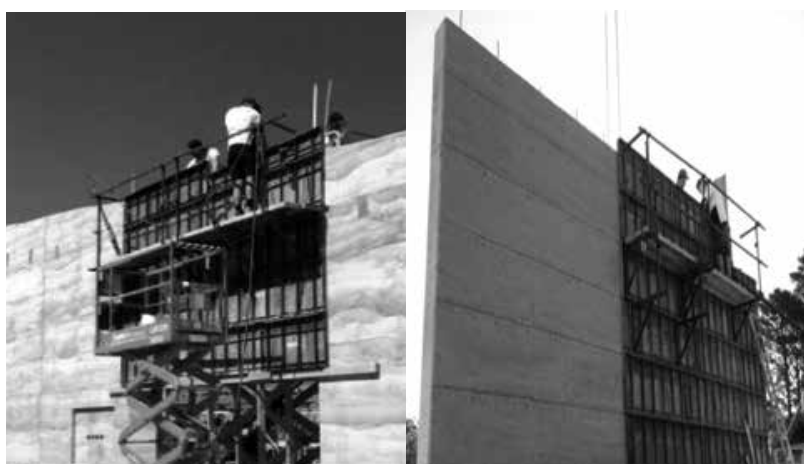

Figura 10. Ejemplos de usos de los moldes y plataformas metálicas apoyadas en cantoneras para ejecución de paredes en tapia pisada. Fuente: Oliver Petrovic y Rodrigo Rocha.

Con relación a las formaletas se sugiere, por tanto, una organización de medidas en múltiplos de $30 \mathrm{~cm}$ para la altura de las paredes buscando finalizarlas dentro de la formaleta; se garantiza así el remate del muro a nivel y en cuanto a la cara de la pared se debe proyectar una diagramación de la posición de las formaletas, siendo ellas sobrepuestas o entrecruzadas, pues su posición interferirá en la apariencia final del muro, como muestra la Figura 10.

\section{Posibilidades de la tapia contemporánea}

En la última década la tapia ha logrado un nivel de elemento de construcción de lujo en Australia, recurrente en las obras de residencias de alto nivel y obras públicas, muy en función de su belleza estética y acabado refinado, así como del costo por metro cuadrado para su ejecución. Pero es importante resaltar que la durabilidad y resistencia estructural, además de la baja o ausencia de mantenimiento de las paredes, son factores que agregan en la elección de los materiales de construcción por los profesionales y clientes o inversores. La diversidad de coloraciones naturales y texturas de las paredes se convierte en uno de los atractivos principales de la aplicación de la tapia en Australia.

Si no se utiliza la tierra del propio local se garantiza la ausencia de materia orgánica en la mezcla, 


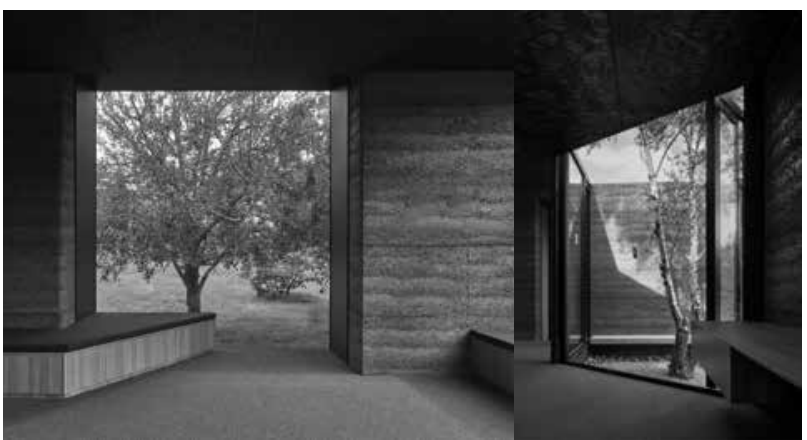

Figura 11. Ejemplos de paredes de tapia en residencia en Australia, mezclada con óxido de hierro, se produce en una coloración grisácea. Fuente: Olnee Constructions.

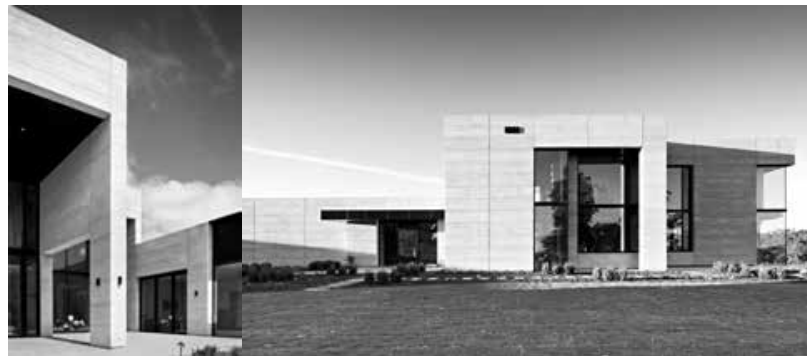

Figura 12. Ejemplos de residencia con paredes de tapia en Australia. Fuente: Olnee Constructions.

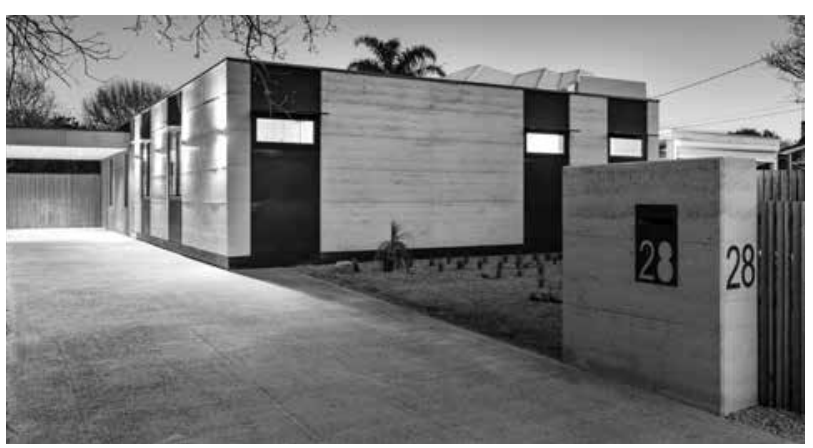

Figura 13. Proyecto de Residencia en Melbourne de autoría del arquitecto Rodrigo Rocha. Fuente: Earth House Australia.

así como el exceso de arcilla y el tamaño de los agregados. A través del mayor entendimiento de los arquitectos e ingenieros sobre la tapia y sus limitaciones, es visto que hay una evolución en el uso estructural y arquitectónico de la tapia en las construcciones contemporáneas, las constructoras de tapia a través de las obras están desarrollando la técnica en sí y perfeccionando la calidad del producto ofrecido.
Hay una expectativa en la sociedad para que la construcción contemporánea se vuelva cada vez más sostenible y minimice el impacto ambiental de su ejecución, así como la extracción, uso y descarte de los materiales de construcción; se cree que la tapia puede desempeñar un papel fundamental en el desarrollo de las construcciones actuales desde el proceso constructivo a la eficiencia energética de las edificaciones.

\section{Consideraciones finales}

La intención de este breve trabajo es la divulgación de una posibilidad de construcción con tierra. Es importante resaltar que el uso del cemento como estabilizante dificulta el descarte de la pared, ya que en una cadena productiva estructurada como la australiana, la pared descartada puede ser triturada y reutilizada como agregado para otras finalidades o incluso para otras paredes con tierra estabilizada.

Se presentaron de forma resumida las características básicas de la técnica constructiva de tapia pisada con tierra estabilizada, reuniendo informaciones y datos que pueden auxiliar en la investigación acerca de las técnicas que utilizan la tierra como material de construcción. En ese sentido, se mostraron ejemplos australianos que poseen una cadena productiva desarrollada para construcción con tapia pisada, desde la mirada del arquitecto que tiene experiencia en el uso de la tapia en Brasil y en Australia, comprendiendo así las necesidades del mercado local y las potencialidades del sistema constructivo en cuestión, proporcionando informaciones constructivas de la tapia de pilón y la manera cómo está siendo desarrollada en la producción arquitectónica contemporánea de Australia.

El suelo, a pesar de ser proporcionalmente el material más empleado en las construcciones en el mundo, en los mercados habituales de países como Brasil aún es clasificado como un material alternativo y primitivo. Todavía gran parte de los arquitectos brasileros duda en utilizar técnicas 
de construcción con tierra en sus proyectos, por no conocer las aplicaciones y posibilidades actuales de uso de este material que se encuentra en abundancia. Por ello, para promover el uso de esta técnica en Brasil se debe fomentar el conocimiento teórico y práctico más allá de proporcionar informaciones técnicas acerca del uso y aplicaciones de este sistema constructivo que une un conocimiento tradicional con el actual desarrollo de la tecnología. Se espera que con este estudio se incentive la investigación y aplicación de esta técnica en el medio académico y profesional, con la intención de ampliar la cadena productiva de las edificaciones que buscan mitigar el impacto ambiental provocado por la industria de la construcción.

\section{Referencias bibliográficas}

Braga Fernandes, M. (2013). A taipa no mundo. Portugal: CEAUCP/CAM Centro de Estudos Arqueológicos das Universidades de Coimbra e Porto/Campo Arqueológico de Mertola

Ciancio, D. \& Beckett, C. (2015). Rammed earth construction, cutting-edge research on traditional and modern rammed earth. Perth, Australia: CRC Press.

Dethier, J. (1986) Des Architectures de Terre. París: Edition de Centre Pompidou.

Dobson, S. (2015). Rammed earth in the modern world. In: Ciancio, D.; Beckett, C. (Eds). Rammed earth construction, cutting-edge research on traditional and modern rammed earth. Perth, Autralia: CRC Press.

Eires, R. y Jalali, S. (2008). Inovações Cientificas de Construção em Terra Crua. Conferência Internacional. Angola: Ensino, Investigação e Desenvolvimento (EIDAO 08).

John, V. M., Silva, V. G. y Agopyan, V. (2001). Agenda 21: uma proposta de discussão para o construbusiness brasileiro. II Encontro nacional e I Encontro Latino americano sobre edificações e comunidades sustentáveis. Canela, Brasil: ANTAC/UFRGS.

Middleton, G. F. (1992). Earth wall construction. Fourth Edition, North Ryde, Australia: CSIRO Division of Building, Construction and Engineering.

Rael, R. (1971). Earth Architecture, Princeton Architectural Press, New York

Torgal, F. P.; Eires, R. M. G. y Jalali, S. (2009). Construção em terra. Portugal: Universidade do Minho, Guimarães.

\section{Agradecimientos}

La gratitud se extiende a todos los que colaboran con el uso e incentivo de construcciones con tierra actualmente, especialmente a Oliver Petrovic por el conocimiento compartido y práctica de más de 30 años construyendo y promoviendo tapias mecanizadas en Australia. Gracias especiales por la traducción al español al arquitecto Juan Manuel Saavedra, Cali, Colombia. 